\title{
CON LOS NERVIOS DE PUNTA: DE LA MEDICALIZACIÓN A LA PSICOLOGIZACIÓN DE LOS QUEBRANTOS MENTALES EN EL TRABAJO. BOGOTÁ Y MEDELLÍN, 1898-1961 ${ }^{1}$
}

\author{
Eugenio Castaño González \\ Universidad de Antioquia \\ Escuela Nacional Sindical \\ Email: eugecas2016@gmail.com \\ ORCID iD: https://orcid.org/0000-0003-3581-7910
}

Recibido: 19 enero 2019; Aceptado: 2 marzo 2020

Cómo citar este artículo/Citation: Castaño González, Eugenio (2020) “Con los nervios de punta: de la medicalización a la psicologización de los quebrantos mentales en el trabajo. Bogotá y Medellín, 1898-1961", Asclepio, 72(2): p319. https://doi.org/10.3989/ asclepio. 2020.20

RESUMEN: En este artículo se analizará el modo en que la neurastenia y la fatiga fueron objetivadas por la medicina dentro del ámbito laboral en las ciudades de Bogotá y Medellín, durante la primera mitad del siglo XX. De ahí la necesidad de tomar como referencia la identificación de los presuntos padecimientos ligados a la esfera del trabajo intelectual y físico. A la reivindicación de los comportamientos frugales para aminorar los efectos de aquellos padecimientos, se le vino a adicionar la creciente psicologización del mundo del trabajo promediando dicha centuria. Aquella psicologización se volvió una herramienta que amplió el espectro de la anormalidad, en medio de la metamorfosis experimentada por el aparato productivo y la diversificación de la estructura laboral a nivel nacional.

PALABRAS CLAVE: Trabajo; Medicalización; Psicologización; Productividad.

\section{WITH THE NERVES ON THE TOP: FROM MEDICALIZATION TO PSYCHOLOGIZATION OF MENTAL BREAK- ERS AT WORK. BOGOTA AND MEDELLÍN, 1898-1961}

ABSTRACT: In this article it will analize the way in which nervous exhaustion and fatigue were objectified by the medicine inside the labour environment in the cities of Bogota and Medellin, during the first half of twentieth century. From there the necessity of taking as reference identification of alleged hardship related to intellectual and physical work sphere. To the demand of frugal behaviours to minimize the effects of that hardship, it added the growing psychologization of world work in the middle of the century. That psychologization was turned into a tool that extended the spectrum of anormality, in the midst of the metamorphosis experienced by the productive system and diversification of labour structure nationally.

KEY WORDS: Labour; Subjectivity; Medicalization; Psychologization; Productivity.

Copyright: @ 2020 CSIC. Este es un artículo de acceso abierto distribuido bajo los términos de la licencia de uso y distribución Creative Commons Reconocimiento 4.0 Internacional (CC BY 4.0) 


\section{INTRODUCCIÓN}

En la primera parte de este artículo se analizará el modo en que ciertas categorías nosográficas como la neurastenia y la fatiga fueron objetivadas desde el ámbito médico, justo en un momento de incipiente industrialización durante el periodo propuesto. También se examinarán las continuidades y discontinuidades alrededor de estos presuntos padecimientos ligados con el mundo del trabajo intelectual y físico. De allí precisamente la pretensión aún incipiente, y en ausencia de una legislación sobre salud laboral referida a los padecimientos mentales ${ }^{2}$, de visibilizarlos desde ciertas disciplinas como la medicina. En segundo lugar, se examinará el paulatino tránsito de lo estrictamente médico a una estrategia de psicologización más amplia, en la medida que la psicología, la psiquiatría y el psicoanálisis se institucionalizaron en Colombia. A ello se le vino a sumar la divulgación de estos discursos en otro tipo de publicaciones no científicas. Lo anterior permite plantear el problema investigativo en torno a cómo se fue configurando una nueva manera de gestionar la fuerza de trabajo, vista como un recurso económico y mental. Se trató de un proceso orientado a la identificación y creciente patologización de comportamientos que presumiblemente ponían en riesgo el desarrollo del aparato productivo.

La periodización elegida responde a dos fenómenos claves. El primero correspondió a la publicación de la tesis de Julio Rodríguez Piñeres en 1898, titulada Neurastenia, en la cual se señala el temor hacia la neurastenia por cuenta de las sobrecargas sociales y laborales, particularmente en lo concerniente al trabajo intelectual. Dicha publicación señala un punto de partida de los procesos de medicalización de lo mental en el mundo del trabajo intelectual. La fecha de cierre corresponde al año 1961, pues durante ese año se creó la Sociedad Colombiana de Psicoanálisis y la Sociedad Colombiana de Psiquiatría. Lo anterior coincidió con la emergencia de una serie de publicaciones en las que el ámbito laboral comenzó a ser examinado desde la psiquiatría, la psicología y el psicoanálisis, y ya no sólo desde la medicina como disciplina hegemónica.

Si bien la extensa temporalidad elegida puede verse como una elección problemática en términos metodológicos, lo cierto es que responde a la intención de examinar, en ausencia de un mayor acopio de fuentes disponibles, la modulación en la forma de objetivar lo mental en el mundo del trabajo; es decir, explorar y analizar el tránsito de la medicalización a la psicologización de lo mental y lo que ello acarreó en el ámbito laboral. En ese sentido, la elección metodológica de los autores de las fuentes consultadas no respondió exclusivamente a la necesidad de referenciarlos como médicos, psiquiatras, entre otros, sino más bien al apremio de problematizar la emergencia y circulación de estos discursos "psi" sobre el ámbito laboral. A ello cabría añadir la ausencia de información y, por consiguiente, la dificultad de realizar un rastreo minucioso de las trayectorias profesionales de cada autor citado en las fuentes primarias, lo cual excede por mucho los alcances y propósitos planteados en este artículo.

A partir de dichas fuentes sobresalen algunos autores, como el ya mencionado médico Julio Rodríguez Piñeres, interno del hospital San Juan de Dios en Bogotá, los médicos Miguel María Calle (empleado de la Empresa Minera del Zancudo), Braulio Mejía, José Miguel Restrepo, Julio Ortiz Vásquez y Eduardo Vasco, docentes de la Universidad de Antioquia. Además de este conjunto de prestigiosos médicos, quienes desarrollaron sus carreras profesionales durante la primera mitad $X X$, es posible identificar a otro tipo de autores, como el Psicólogo social y sacerdote Ireneo Rosie, el Psicólogo y Antropólogo social Enrique Valencia y el Psiquiatra Luis Gabriel Jaramillo. Más allá de dichas trayectorias individuales, interesa más destacar la creciente objetivación de ciertos padecimientos emocionales en publicaciones de origen médico durante la primera mitad del siglo XX (además de las tesis), como el Boletín Clínico. Órgano de la Facultad de Medicina y Ciencias Naturales de la Universidad de Antioquia; Anales de la Academia de Medicina de Medellín; Orientaciones médicas. A lo señalado acá cabría agregar, por un lado, otras publicaciones especializadas, como la Revista Colombiana de Psiquiatría, fundada en 1964, la cual puso de relieve el paulatino tránsito que se desea problematizar en el presente artículo. Y, por otro lado, algunos artículos de revistas no especializadas y magazines, como El Correo de Medellín y la Revista Cromos de Bogotá.

A ello se le añade la necesidad de articular lo anterior con algunos cambios socioeconómicos experimentados durante el periodo propuesto, con el propósito de realizar un análisis histórico más profundo. Por lo tanto, lo abordado hasta ahora no se orienta a dilucidar la maquinaria moral-religiosa destinada a reconducir los comportamientos de las clases traba- 
jadoras, por causa de la pobreza y las pésimas condiciones socio-ambientales (Foucault, 2000, p. 294). Lo que subyace es una perspectiva diferente a aquella vinculada con la teoría de la degeneración racial y la moral religiosa, pero sí más ajustada, a partir de la segunda posguerra, a la psicologización del proletario y los movimientos organizativos. Si bajo los principios deterministas el ser humano era concebido como una máquina movida por el espeso combustible de las circunstancias biológicas y la herencia, el aspecto psicológico, según escribía Prospero Rueda, en la revista Temas en el mes de enero de 1944 , estaba sujeto a la personalidad, abordada desde lo individual y lo social ${ }^{3}$. Como telón de fondo se hallaba, por un lado, la creación del ya mencionado grupo psicoanalítico en Colombia, su reconocimiento internacional en 1959 con la consiguiente organización del Instituto Psicoanalítico ese mismo año, además de la creación de la Sociedad Colombiana de Psicoanálisis en 1961; pero, por otro lado, se observó un contexto social caracterizado por la consolidación del Frente Nacional en Colombia y el fortalecimiento del movimiento obrero durante la segunda mitad del siglo XX (Archila, 2003a, p. 508). Fue este periodo de auge del dispositivo industrial el que consolidó un imaginario del movimiento obrero como objeto de sospecha y presunto enemigo del progreso dentro de ciertos círculos económicos, políticos e intelectuales (Castaño, 2015b, p. 482).

Por otro lado, la elección de las ciudades de Bogotá y Medellín para el rastreo de fuentes obedeció a tres motivos: el primero, porque estas ciudades, desde finales del siglo XIX y comienzos del XX se constituyeron en los principales centros urbanos del país. En segundo lugar, porque ambas se establecieron como los principales núcleos del proceso de industrialización durante todo el siglo XX en Colombia. En tercer lugar, y partiendo de los dos motivos anteriores, así como de las fuentes disponibles para el desarrollo del presente artículo, estas dos ciudades se erigieron en los primeros y principales centros de divulgación de saberes sobre los procesos de medicalización y psicologización de los trabajadores colombianos, lo que no significa que fuesen los únicos. La disparidad, heterogeneidad y escasa disponibilidad de las fuentes tornó difícil examinar ambas ciudades como estructuras analíticas separadas dentro del artículo; aun cuando, en la medida de lo posible, se evitó establecer generalizaciones con alcances nacionales en las interpretaciones realizadas.
Es preciso aclarar que este artículo no se ajusta a un enfoque ligado con la historia de la disciplina médica, psiquiátrica o psicológica. Tampoco se pretende realizar un análisis epistemológico de la fatiga o la neurastenia, lo cual ya ha sido abordado de manera más detallada por otros autores (Vásquez, 2015). Para efectos del presente artículo, se desea abordar y reproblematizar dichas dolencias, sus continuidades $y$ discontinuidades, a la luz de los excesos en los trabajos intelectuales y físicos desempeñados durante el periodo analizado. Para esto, se examinó en la primera parte del artículo la forma en que dichos padecimientos fueron objetivados como enfermedades desencadenadas por las características del trabajo intelectual. Con ello también se pretende explicar los potenciales riesgos que ofrecía para la psique un trabajo mal encauzado, en medio de entorno urbanizado y con un aparato productivo más diversificado. Se trata de dar cuenta de la forma cómo la realidad del mundo del trabajo comenzó a ser considerada en función de los desequilibrios mentales que era capaz de acarrear, lo cual hizo parte de una estrategia de racionalización y optimización de la fuerza productiva.

Tomando en consideración lo anterior, al revisar la producción historiográfica a nivel internacional sobre la relación histórica entre el mundo del trabajo y la objetivación de lo mental, es posible identificar algunos enfoques investigativos. El primero de ellos, desarrollado por autores como Saraceno (2013), Billiard (2002), Ouvrier (2010), Le Bianic (2004) y Vatin (2004), Rabinbach (1990), se centra en el análisis de la sociedad salarial europea y norteamericana del siglo $X X$. Este enfoque examina cómo se desarrolló el estudio de la actividad corporal y mental de los trabajadores desde una perspectiva psicofisiológica. Para ello abordan el asunto del cuerpo como motor, la orientación, selección y reclutamiento de obreros durante la primera mitad del siglo $\mathrm{XX}$, con el objetivo de determinar las aptitudes laborales desde la psicología experimental. Si este primer enfoque vuelca la atención en la primera mitad del siglo XX, los estudios sobre la gubernamentalidad desarrollados en el ámbito anglosajón, en cabeza de Nikolas Rose (1990, p. 352), se ocupan de la implementación de prácticas de subjetivación laboral en el ámbito europeo y norteamericano durante el siglo XX y XXI, partiendo de ciertos discursos psicológico-administrativos. En el ámbito latinoamericano cobran un valor de capital importancia los trabajos de autores como Gallo (2016a, 2016b), Valero (2009, 2010, 2014), Caponi 
(2015), Haidar (2016), Roldán (2010), Motta (2008), Ruperthuz (2019), Gallo \& Ramacciotti (2019), y Jaraba (2014). Con base en las pesquisas desarrolladas por estos autores es posible identificar tres ámbitos de trabajo muy vinculados.

El primero corresponde a la exploración de fenómenos como la fatiga en el ámbito laboral y sus formas de objetivación durante la primera mitad del siglo XX. Se trata de un tipo de análisis histórico que pone de relieve -en un contexto de creciente industrialización -la intención de regular los cuerpos para reformar las mentes con el fin de optimizar rendimientos. Un segundo ámbito de trabajo se desprende de las pesquisas históricas sobre el concepto del factor humano, vinculado con la emergencia de la fatiga, el estrés, además de otros fenómenos como la psicotecnia, el conductismo y el psicoanálisis. Lo anterior procuró dar un paso adelante en ese proyecto de psicologización del ámbito laboral, tal como se puede examinar en Ruperthuz (2019) para el caso chileno y de los análisis del cuerpo humano desde la metáfora del "motor", tal como lo plantea el colombiano Valero. El tercero se enfoca en el problema de la simulación. Se trata de resaltar el rol desempeñado por la psiquiatría francesa, la práctica médico-legal y la medicina del trabajo en la objetivación de este padecimiento en situaciones de accidentalidad laboral (GaIlo, 2016 a). Existe un punto en común entre estos tres ejes investigativos en torno al esclarecimiento de los mecanismos orientados a la optimización de los rendimientos laborales desde el plano emocional, guiados por una experiencia de reingeniería mental en las sociedades modernas y contemporáneas.

Si el anterior balance refleja una escasa atención por estos temas como fuente de reflexión historiográfica en el plano internacional, en el plano nacional esta tendencia es mucho más notoria. A pesar de que Gallo ha explorado el fenómeno de la fatiga, la simulación y el factor humano, sus pesquisas, por un lado, se han ubicado temporalmente en la primera mitad del siglo XX; y, por otro lado, su principal objeto de estudio ha sido el análisis de la legislación laboral en Colombia, las enfermedades profesionales y las prácticas médicas, especialmente en zonas como la antigua Mina del Zancudo, ubicada en el departamento de Antioquia. De allí se desprende que, a la escasa inquietud por emprender investigaciones históricas situadas en la segunda mitad del siglo XX, se le suma la atención inexistente de la historiografía colombiana por examinar en profundidad estos discursos psico- lógicos dentro del ámbito laboral. Es frente a esto último que cobra relevancia el presente artículo, en la medida que pretende centrar la atención en dichos vacíos, enfocándose específicamente en el punto de intersección entre lo médico y lo psicológico.

\section{LA NEURASTENIA Y LOS EXCESOS CIVILIZATORIOS}

Si por un lado el control sobre el tiempo de los demás se convirtió en un mecanismo fundamental para hacer más fluida y adaptable la mano de obra, por otro lado, el factor tiempo en el trabajo se tradujo en una creciente presión psicológica y física que obligó a los individuos a adaptarse a un ritmo cada vez más vertiginoso (Hopenhayn, 2001, p. 149). Los efectos mentales de la dilatada vida urbana comenzaron a ser objeto de preocupaciones. En 1881 George Miller Beard identificó la vida moderna con la neurastenia en el American Nervousness, al igual que Adrian Proust, autor francés del manual sobre neurastenia L'higiene du neurasténique (Rabinbach, 1996). Ambos autores concebían la neurastenia como un padecimiento que tendía a predominar en las ciudades y sus intensos ambientes, particularmente entre la clase media y alta, y en medio de la cultura intelectual. Para el primero de ellos, el análisis de esta enfermedad tenía como referencia el equilibrio de la energía nerviosa de los individuos, por lo que existían personas con muy pocos recursos energéticos y otras a quienes les sobraba. Se afirmaba que los neurasténicos mostraban con frecuencia una extrema falta de voluntad, cansancio, abulia, lo cual se veía reflejado en su desidia hacia el trabajo. Esta ausencia de voluntad, según Théodule Ribot en su Les Maladies de la volunté, reseñada por Rabinbach (1996), era susceptible de padecerse debido al estilo de vida de las clases más acomodadas, particularmente las mujeres con una posición económica solvente. La evocación de estos padecimientos despertó las alarmas de la Europa decimonónica, pues la resistencia a la ética laboral, a la productividad y a la prodigalidad del trabajo duro, se tornaba en una amenaza a los valores existentes (Rabinbach, 1996).

Para el caso colombiano, la referencia más antigua que se tiene sobre el tema se halla en el texto del italiano Pablo Mantegazza, traducido por Baldomero Sanín Cano en el año 1888 y publicado por la Imprenta La Luz en la ciudad de Bogotá4. Este libro es bastante interesante porque, en principio, pone de re- 
lieve una relación inextricable entre los discursos de la pastoral católica con ciertas premisas médicas, a la hora de abordar el problema de la neurosis. La mezcla entre ambos postulados da cuenta de la manera cómo el concurso de estos principios religiosos, a tan solo un año de haberse firmado el concordato entre el gobierno de Colombia con la Santa Sede, respondía a la intención de brindar legitimidad a un nuevo campo de saber sobre lo mental. De manera que la previsión económica, la virtud ligada a la prudencia en el gasto y la pericia para adentrarse en los sucesos del futuro, propio del individuo cuerdo, trabajador y acomodado, limitaban las posibilidades de ser víctima de la neurosis ${ }^{5}$. Es llamativo constatar el contraste entre este arquetipo del burgués previsor y metódico, con la descripción realizada por Mantegazza sobre un individuo dedicado al periodismo. El contraste entre el hombre trabajador, consagrado a los negocios, y el periodista que despilfarraba su dinero y su tiempo para sucumbir luego ante la neuro$\mathrm{sis}^{6}$, se erigió, en este caso, en un modelo de conducta para afrontar los desafíos impuestos por el siglo venidero.

Esta forma de definir la neurosis como la enfermedad del siglo, tuvo una enorme influencia en el vasto aparato teórico desarrollado por autores nacionales como Lázaro Escobar, según Roselli (1968), y por el médico bogotano Julio Rodríguez Piñeres (1898). Diez años después de traducido el texto de Mantegazza, el 10 de noviembre de 1898 se publicó en Bogotá la tesis de grado en medicina y cirugía de Piñeres, titulada Neurastenia ${ }^{7}$. La importancia de dicho documento reside en que, probablemente, fuese el primer texto escrito por un colombiano en el que se estudiaba en detalle el fenómeno de la neurastenia y su relación con el mundo laboral. Si bien el documento traducido por Sanín Cano hacía alusión a la neurosis o neurosismo, la publicación del médico bogotano hacía una mención más directa y precisa al concepto de neurastenia o neurosis neurasténica. Según él, y en correspondencia con lo afirmado por el autor italiano unos años atrás, esta forma de neurosis denominada neurastenia se expresaba a través de un abuso de las sensaciones, el sobrecargo permanente del cuerpo y el espíritu por el trabajo intelectual que, articulado con la predisposición hereditaria, era capaz de excitar el sistema nervioso. El temor frente a la capacidad desestabilizadora de los centros urbanos, sumado a las posibles insuficiencias hereditarias de los individuos, despertaba una enorme expectativa por el futuro más próximo del país y su capacidad de producción ${ }^{8}$.

El cruce entre los desarrollos teóricos sobre la raza, el trabajo y los estilos de vida (Solano, 2011; Villegas, 2014), se establecieron en elementos claves para comprender este tipo de afirmaciones. De igual forma, el querer ir más allá de sus posibilidades inmediatas, la tenaz lucha por hacerse un lugar respetable en los lugares de trabajo, el deseo de sobresalir laboralmente en un ambiente colmado de estímulos mundanos, instauraba en la mente de los individuos una sobrecarga general en el sistema nervioso. Para Rodríguez Piñeres (1898), igual que Mantegazza, en los grandes trabajadores del pensamiento, como los escritores o entre quienes se sumergían en la vorágine de los negocios, el comercio, la política, la medicina, el ejército ${ }^{9}$, la docencia, se podía constatar verdaderos raptos de depresión cerebral ${ }^{10}$.

\section{LOS OBREROS TAMBIÉN SUFREN}

Si para ambos la neurastenia no parecía aquejar a las profesiones que requerían poco esfuerzo intelectual ${ }^{11}$, otros autores de la época desarrollaron una visión diferente sobre los efectos del trabajo mental en los obreros. Por ejemplo, Miguel María Calle, médico de la empresa minera del Zancudo, ubicada en el departamento de Antioquia, escribió un artículo en el año 1910 titulado "Astenia de los obreros"12. Allí afirmaba que la categoría neurastenia, que definía un sinnúmero de manifestaciones mórbidas, estaba siendo reemplazada por la categoría astenia, la cual abarcaba distintas modalidades de agotamiento físico y psíquico. La relación entre las precarias condiciones sociales y aquel padecimiento, trajo aparejado una connotación mucho más estrecha en relación con la pobreza y con enfermedades orgánicas como la tuberculosis, gripa y albuminuria ${ }^{13}$. Así, a diferencia de la neurastenia, que parecía aquejar a un sector poblacional privilegiado y vinculado con los trabajos intelectuales a comienzos de siglo, la astenia se describía como un padecimiento que afectaba a un sector poblacional mucho más amplio y ubicado en la base de la pirámide social ${ }^{14}$. Esta situación debía poner a los galenos frente al reto de analizar cómo los acontecimientos políticos sucedidos en Colombia durante aquella época, habían contribuido a deteriorar las condiciones fisiológicas y mentales de los jornaleros más desfavorecidos ${ }^{15}$. Antes de la guerra de los mil días, según él, había mejores salarios y los artí- 
culos de primera necesidad no ostentaban, al parecer, los precios escandalosos que se vislumbraban en el año en el cual se escribió el artículo. Por tal razón, la reivindicación de un pasado bucólico contrastaba con la visión pesimista presentada por Sanín Cano en su traducción del libro de Mantegazza (1888). Si en este último se vislumbraba un nuevo futuro con el cambio de centuria, quizás más promisorio, que dejaba atrás las incidencias nefastas del siglo XIX sobre la psique de los trabajadores europeos ${ }^{16}$, en el planteamiento de los autores colombianos se invertían los términos del análisis para dar cuenta de la crítica situación nacional.

Dicho de otra forma, si la neurastenia parecía replegarse en el ámbito europeo, de acuerdo con el planteamiento del autor italiano, para los autores colombianos como Calle (1910), sucedía todo lo contrario. Para este último, por ejemplo, era necesario volcar la atención sobre las transformaciones culturales, económicas y sociales que experimentaba el país durante el cambio de siglo. Con ello, según él, se dejaba atrás un periodo caracterizado por un estilo bucólico y carente de estímulos para la psique humana, en espera de ingresar a una nueva época más agitada ${ }^{17}$. A finales de siglo no existía un proyecto consolidado de economía nacional integral, lo cual se veía reflejado en la fragmentación regional y el escaso desarrollo de la infraestructura industrial (Sánchez, 2009). Así mismo, a pesar de que la política proteccionista iniciada por el presidente Rafael Reyes, a comienzos del siglo XX (1904-1909), tuvo una influencia decisiva en la industria de los ladrillos, las gaseosas, empresas textileras, además de la ampliación de algunas vías férreas, la incidencia del sector industrial en la economía nacional seguía siendo marginal. Por tal razón, dar vida a la nación industrial soñada por los letrados del siglo XIX y comienzos del XX conllevaba superar las barreras geográficas a través de la implementación de medios de transporte capaces de acortar las distancias entre las regiones, y de estas con el mundo (Kalmanovitz, 2015, p. 245).

No obstante, el aumento de tamaño de ciudades como Bogotá y Medellín (Castro, 2009, p. 274), el incremento de la velocidad en los desplazamientos por los nuevos medios de transporte como el tranvía y los ferrocarriles, el mejoramiento en el sistema de alcantarillado e iluminación, fue el reflejo de esa mixtura entre aquella comunidad de trabajadores intelectuales decimonónicos, con los artesanos, comerciantes y aquellos otros vinculados con la incipiente economía industrial. Como telón de fondo de este lento proceso de industrialización yacían las denuncias por la presunta pérdida de la rectitud moral y la avidez de la nueva economía industrial en ciernes. Este frenesí se proyectaba en el anhelo de emprender fantásticas empresas de minas, ferrocarriles y bancos. Para Basilio Uribe, detrás de ello reinaban una serie de personajes salidos del quicio, individuos que emigraban a la ciudad con deseos desbordados de reconocimiento y obtención de méritos laborales que creían merecer, convirtiéndose en árbitros para todas las cuestiones políticas, sociales y económicas. Así, todo aquel que pasaba los muros de ciudades como Medellín y Bogotá, era confundido, arrastrado por la multitud y por aquella atmosfera caldeada por la neurosis y el servilismo ${ }^{18}$.

Cabe destacar que dichos señalamientos no ponían en cuestión el valor supremo del trabajo por las riquezas que proveía, o bien por su capacidad redentora. Existía un cierto principio moral ligado al desarrollo del mundo moderno, en donde el trabajo se fue estableciendo en el soporte cotidiano del vínculo social ${ }^{19}$. Todas las facultades eran vistas como poderes activos que debían ponerse en marcha por medio de la acción permanente, una especie de engranaje que requería de una vigorosa fuerza propulsora ${ }^{20}$. Algunos rotativos que circulaban a comienzos de siglo, como el caso del periódico El SOL ${ }^{21}$, hacían de la experiencia y el gusto por la vida un elemento inextricablemente unido al trabajo arduo. La vida en ocio era vislumbrada como una ausencia, una carencia de fibra vital para el alma, un instrumento de acero en estado de lenta e inexorable oxidación.

En resumen, la representación de la vida moderna de comienzos de siglo, caracterizada por el creciente proceso de diferenciación social y laboral, aunque muy incipiente para el caso colombiano, promovió un debate alrededor de padecimientos mentales asociados al torbellino ofrecido por el trepidante ritmo del trabajo intelectual. Sin embargo, visto desde el otro extremo, la ausencia de actividad también fue concebida como un elemento capaz de alterar el equilibrio psíquico, además de atentar contra el "progreso nacional"22. Incluso, a mediados de siglo, Mora (1949) afirmaba que uno de los riesgos en la iniciación en la vida del trabajo manual, arduo, disciplinado y constante, era la educación mal orientada. De modo que, si el trabajo intelectual se veía como un desencadenante de padecimientos psíquicos, también se representó, para el caso específico de las cavilaciones lite- 
rarias, como una actividad poco productiva derivada de una mala orientación en las instituciones educativas (Mora, 1949). A ello cabría añadir que la crisis económica por la que atravesó el país durante la década del treinta, fue vista como un fenómeno que dio lugar a presuntos estados patológicos durante los años siguientes ${ }^{23}$. A la exigente vida de los abogados, médicos, profesores, soldados, literatos y sacerdotes, entre otros, se le añadieron, a partir de la tercera y cuarta década del siglo $\mathrm{XX}$, los señalamientos cada vez más recurrentes contra la población obrera y los empleados $^{24}$. El incremento de los dispositivos fabriles y, por ende, la mayor representatividad del obrero dentro de la estructura productiva del país, puso en juego una atención más marcada por los conflictos y los peligros suscitados dentro de los lugares de trabajo. En términos generales, no solo la lectura, la comodidad, los placeres y el reposo físico en favor de las labores intelectuales, despertaron las alarmas por lo que pudiesen provocar. El sufrimiento y las penurias $^{25}$, el sometimiento a otros entornos malsanos y hostiles ${ }^{26}$, el abuso de estimulantes y enfermedades como la influenza ${ }^{27}$, además de las características propias del trabajo obrero, también fueron vistos como potenciales elementos desestabilizadores de la psique.

\section{LA FATIGA Y EL PELIGRO DE LA IMPRODUCTIVIDAD}

La paulatina diversificación de la estructura laboral en el país también tuvo su correlato en la objetivación de las carencias y los esfuerzos realizados por los trabajadores, a través de una red de padecimientos como la fatiga mental. El anhelo por escudriñar los desórdenes espirituales a finales del siglo XIX y durante el transcurso de la siguiente centuria, así como la búsqueda por clasificar comportamientos y sufrimientos, no sólo se tradujo en la objetivación de categorías nosológicas, sino que también puso en marcha toda una batería sintomatológica estrechamente atada a la neurastenia, como fue el caso de la fatiga. Esta última se asoció con los desórdenes modernos, enfermedades ligadas a la industria, algunas de ellas supeditadas al desgaste de energía y a la propia neurastenia. Estos puntos de análisis marcaron una diferencia en relación con lo planteado por la neurastenia. Si en esta última el motivo del desarreglo mental estribaba en la desmesura experimentada por ciertas acciones introspectivas y reflexivas, ligadas a la vorágine "civilizatoria" y al trabajo inmaterial de comienzos de siglo, la fatiga, orientada hacia un enfoque psicopatológico (Vatin, 2004, p. 244), pareció establecer una relación un poco más estrecha con una franja poblacional ubicada en la base de la pirámide social.

Así, en 1937 el médico Emilio Morales hacía mención a la monotonía en el trabajo y la reacción del individuo, ligado a la fatiga, además de señalar el vínculo existente entre la fatiga y la neurastenia. Tal como lo menciona Gallo, Morales abordaba el problema de la fatiga a partir de la fisiología del trabajo o fisiología industrial, esta última asociada con una perspectiva psicosocial (Gallo, 2016 a). Dicha perspectiva parecía tornarse cada vez más visible en diferentes ámbitos laborales, como los trabajos de oficina. El escritor bogotano J. A. Osorio Lizarazo realizaba una sombría descripción de las labores realizadas en aquellos sitios:

Todas las mañanas, cada pieza, cada empleado, se inclina sobre los papeles y penetra dentro de la rutina. Una perezosa rutina de laxas esperanzas, de tibias ejecuciones, de limitadas y problemáticas perspectivas, dentro de la cual se va diluyendo la personalidad, perece la iniciativa y se asfixia el individuo. La espontaneidad ha perecido. Después se extiende una ficción de trabajo, en la cual cada uno trata de dar una sensación de laboriosidad, cuando en realidad sólo espera con impaciencia la hora de salida o el día de fiesta ${ }^{28}$.

A lo señalado por Lizarazo en la anterior cita se le añaden otros factores mentales para analizar asuntos como la experiencia del trabajador, el interés, el grado de responsabilidad, el temor, la emoción, la ansiedad y la pena. De manera que las condiciones socio-espaciales, además de los hábitos del trabajador, jugaron un papel importante en los planteamientos de Morales en aspectos puntuales como las obligaciones con la familia y el país, algunos excitantes como el alcohol y el tabaco, las relaciones sexuales, la distribución espacial de las viviendas, la distancia entre los hogares y el trabajo, la incomprensión del trabajo, los excitantes de velocidad, la actitud mental frente a la dirección de la empresa o fábrica, los sentimientos religiosos, patrióticos, entre otros ${ }^{29}$. El carácter individual también entrañaba una serie de rasgos morales y sociales cuestionables, tales como actitudes negligentes, inquietas, indiferentes, que buscarían excitaciones mal sanas capaces de alterar el orden establecido $^{30}$. Los reflejos sociales de la fatiga mental promovieron la alerta sobre el riesgo de un nuevo tipo de enfermedad social, expresada a través de la 
desconfianza y la nefasta improductibilidad de las masas $^{31}$.

De allí que esta preocupación por las consecuencias nefastas de aquellos dolencias derivaron en que, a partir de la segunda mitad de la década del cuarenta, el ingeniero e inmigrante español César de Madariaga comenzara a prestar atención sobre los efectos de la mencionada monotonía en el sistema nervioso, recomendando el reposo como forma de aminorar los impactos sobre la psique de los trabajadores colombianos $^{32}$. El objetivo de ponderar y maximizar el tiempo laboral se constituyó en una estrategia orientada a delimitar unos perfiles psicológicos y ocupacionales requeridos por los empresarios de la época. Lo anterior se llevó a cabo en medio de un previo contexto histórico, caracterizado por una creciente mixtura entre aquella comunidad de trabajadores intelectuales decimonónicos, con los artesanos, comerciantes y otros vinculados con la incipiente economía industrial que empezó a despuntar durante las primeras décadas del siglo XX. El arribo de los gobiernos liberales de la década del treinta, comenzando por Enrique Olaya Herrera (1930), se articuló con un crecimiento significativo de la industria.

Las élites fueron abandonando el modelo de libre cambio por uno de mayor intervención estatal, lo cual se vio expresado en que para la década del treinta el proceso de industrialización, concentrado fundamentalmente en bienes de consumo, ya no fuese un fenómeno tan poco usual como a comienzos de siglo (Archila, 2003b, p. 475). De modo que, si durante las primeras décadas del siglo XX se experimentó un crecimiento de la industria nacional, a partir de la década del cuarenta y, especialmente, luego de la segunda posguerra, se acentuó dicha tendencia a través de un proceso más consolidado de sustitución de importaciones y de diversificación del mundo laboral. La intervención estatal, producto de las restricciones del flujo comercial ocasionado por la guerra, se hizo mucho más notoria en varios aspectos económicos, entre ellos el otorgamiento de subsidios directos e indirectos a empresas industriales que generaran empleo (Ocampo et al., 1987).

Precisamente, a partir de aquel periodo, caracterizado por aquellas transformaciones anteriormente mencionadas, se hizo mayor énfasis en la necesidad de alejar las preocupaciones y la indisciplina mental, capaces de alterar las funciones del organismo y hacer menos eficientes a los individuos en la industria ${ }^{33}$. La relación entre el ejercicio de una profesión o una labor determinada, con posibles estados mentales, permitió valerse de todo un caudal teórico orientado a describir, analizar, clasificar y prescribir comportamientos aparentemente patológicos que pondrían en riesgo la producción del país. La búsqueda de constantes objetivas como parámetros de medición y clasificación de lo normal y lo anormal (Canguilhem, 1986, p. 242) en el ámbito laboral, comenzó a valerse cada vez más del recurso a una idea de regularidad global y vital. Lo normal fue entendido por autores como Eduardo Vasco ${ }^{34}$, a finales de la década del cuarenta, de manera semejante a lo desarrollado por Quetelet y Durkheim (2008), tomando como base los planteamientos estadísticos y la determinación de frecuencias. La propuesta de Vasco consistía en concebir lo normal como promedio existente y como modelo de perfección (Hacking, 2006, p. 242). No cabe duda que ello entrañó una producción de valoraciones sobre entornos y conductas cada vez más complejas que las del simple neurasténico de comienzos de siglo y luego del aquejado de fatiga. Se trataba con ello de trazar otro tipo de rumbo vital de los seres humanos en sus rendimientos laborales, en términos de diferencias y deficiencias. El autor traía a cuento la capacidad intelectual del individuo y su correlación con la respectiva capacidad productiva ${ }^{35}$, por lo que la detección previa de un estándar "normal o anormal" de desarrollo psíquico en la niñez dentro de la escuela, debería de servir para pronosticar y encasillar los futuros niveles de éxito o de fracaso en los lugares de trabajo. A este hecho se le añadía la necesidad de enaltecer los factores de orden afectivo y ambiental del niño, como ingredientes determinadores del futuro éxito o fracaso en el desempeño de los roles ciudadanos y laborales.

De lo anterior concluía que el niño superdotado sería capaz, en un futuro, de producir para él y su familia, podría ahorrar y ejercer influencia sobre los demás. El niño normal produciría para él y su familia, asegurándose el porvenir a través del ahorro. El definido como lerdo podría sostener una pequeña familia, pero no estaría en capacidad de entender la importancia de la economía para el futuro. El débil mental alcanzaría a ganar su propio sostenimiento. El imbécil podría producir media ración y la otra media se la tendría que dotar la beneficencia o bien a través de medios ilícitos. Por último, el idiota sería carga social. Dichas conclusiones serían aceptadas favorable o desfavorablemente por las líneas generales del carácter, pues un imbécil, disciplinado y de buena índole, estaría en capacidad de ganar su ración 
completa; y, por el contrario, un intelectual, ya fuese por condiciones de temperamento, de desadaptación o por las propias condiciones ambientales, podría llegar a convertirse en un valor negativo para la sociedad en que residía ${ }^{36}$. Esta necesidad de identificar, patologizar comportamientos, además de buscar optimizar rendimientos, coincidió con la creación del Instituto de Fomento Industrial en 1940 por el entonces presidente Eduardo Santos. A ello se le añadió que la segunda pos guerra dio paso a la consolidación de otras actividades económicas como el transporte, sector financiero, comunicaciones, servicios públicos modernos. Lo anterior se tradujo en el incremento del trabajo asalariado (distribuido en peones, obreros y empleados) entre finales de la década del treinta y comienzos de la del sesenta (Sánchez, 2009, p.173). En efecto, según Ocampo, el sector servicios acrecentó su participación en la generación de ocupaciones del $21 \%$ en 1938 al $45 \%$ en 1984 (Ocampo et al., 1987).

\section{EL MIEDO AL PROLETARIO Y LA PSICOLOGIZACIÓN DE LO MENTAL}

La diversificación del aparato productivo y del trabajo asalariado puso en juego una creciente y compleja atención hacia la psique en las relaciones laborales, como fuente de productividad. Por ejemplo, en otro tipo de magazines, como la revista Cromos, se describieron las tipologías de lo que allí referían como "homeotermos degenerados" 37 , caracterizados por su incapacidad para restablecer las maquinarias biológicas y psíquicas. Si la falta de interés se asoció con el rigor de ciertas condiciones deficitarias, propias del entorno del individuo, o bien dentro del individuo mismo, lo que pregonaron otras publicaciones como Cromos, por el contrario, era que la comodidad misma se había constituido en el fermento propicio de la fatiga ${ }^{38}$. La característica de este fenómeno, según el artículo de Cromos de finales de la década del cincuenta, radicaba en lo errado de intentar explicar la manifestación de la fatiga como efecto del exceso de trabajo:

Muchas gentes se quejan de que sus trabajos los están matando. Pero es difícil imaginar una tarea que cuadre realmente con esa descripción. No es el trabajo el que afecta la energía emocional de la persona. Cuando está descontento con su trabajo o sufre de presión imaginaria o real de sus superiores, excusa la falta de entusiasmo en la tarea con la queja de que el trabajo es excesivo, enfermizo y matador. Esto es injusto no sólo hacia el patrón, sino también hacia la familia ${ }^{39}$.

Aquella publicación puso en evidencia la necesidad de incorporar un régimen de vida capaz de conminar los efectos mentales de la fatiga, sin menoscabar con ello los intereses productivos, en plena etapa de sustitución de importaciones. Si durante la primera mitad del siglo XX la neurastenia y la fatiga presuntamente se desencadenaban por la sobrecarga laboral y un entorno social vertiginoso, a partir de la década del cincuenta la sospecha también recayó en la comodidad que ofrecía la ausencia de trabajo, o una labor poco o nada estimulante. Esta preocupación por las manifestaciones mentales de la fatiga, no solo invirtió la escala de valoración sobre el régimen recomendado para combatir la neurastenia, sino que también reveló un creciente temor sobre los efectos desestabilizadores en los asalariados. A los trabajadores colombianos se les exigió adecuarse a las necesidades de unos empresarios privados, quienes exigían docilidad, disciplina, productividad y mucha fe. ${ }^{40}$ La cantina, los cafés, el billar, el cine y la vagancia también ofrecieron una imagen de decadencia espiritual, de suciedad e "infección" psicológica para los trabajadores ${ }^{41}$. La relación inextricable entre el entorno social y laboral evidenció una representación más alarmista que a comienzos de siglo, poniendo de relieve el supuesto incremento de las patologías mentales en Colombia durante la década del sesenta y setenta ${ }^{42}$. Esta mayor atención a los padecimientos psíquicos tuvo una relación estrecha con varios acontecimientos claves, como fue la creación de la Facultad de Psicología de la Universidad Nacional en 1947, el establecimiento de la Sociedad Colombiana de Psicoanálisis y la creación de la Sociedad Colombiana de Psiquiatría en 1961 (Roselli, Otero y Heller, 2000).

La emergencia de nuevas dolencias hizo apremiante la necesidad de intervenir los presuntos desarreglos mentales de ciertos individuos, con el apoyo de otras especialidades como la endocrinología, neurología, cirugía, cardiología, la psiquiatría y la psicología. Se definieron nuevos síndromes y cuadros clínicos, lo cual obligó a ampliar la atención sobre el ámbito laboral y lo que podría acarrear. Entre dichos síndromes destacaban las neurosis de las telefonistas, de los pianistas, los calambres de los escribanos y de los mecanotaquígrafos, la hipocondría de las secretarias, las obsesiones de los dibujantes, los complejos de ejecutivos que podrían desembocar en úlceras pépticas o colitis ulcerosas ${ }^{43}$. Los conglomerados fabriles 
también ocuparon un espacio como proveedores, no sólo de oportunidades de realización laboral, de plenitud individual y familiar, sino que también fueron señalados de desencadenar nuevas dolencias, indisposiciones, perturbaciones en mentes que presumiblemente jamás tendrían sosiego ${ }^{44}$.

Por otro lado, si en los planteamientos de autores como Uribe Cualla se prestó atención al fenómeno de la inmigración como medida de salvaguarda para conservar las dotes mentales de los individuos aptos para el trabajo en las ciudades colombianas ${ }^{45}$, lo que se observó luego fue un detenido escrutinio de la personalidad del obrero incorporado a los complejos engranajes sociales y productivos. No se trataba solo de develar los secretos y oscuros problemas psíquicos ocasionados por la ciudad, por el trabajo intelectual en el caso de escritores, maestros, periodistas, entre otros, tal como se apreció a comienzos del siglo XX. En este caso, se trataba de identificar un cortejo de sufrimientos psicológicos asociados con las propias dinámicas del trabajo asalariado en expansión. De manera que asuntos como las relaciones interpersonales, la inconformidad con el salario, el disgusto por el ascenso de los compañeros a quienes no se estimaba, la dureza de algunos jefes para ordenar y hacerse obedecer, también fueron objetivados como estímulos desencadenantes de estas supuestas dolencias psíquicas ${ }^{46}$

En un artículo escrito a finales de la década del sesenta por Restrepo, Jaramillo, Valencia y Rosie, se señalaba que tan sólo al hablar en términos amistosos con los proletarios y al analizar sus palabras "explosivas", era suficiente para comprobar sus expresiones anómalas. Por ello era necesario ampliar la capacidad de lectura del comportamiento humano para adentrarse en los máximos reductos de la intimidad. En este tipo de abordajes las posiciones racialistas parecieron dejar paso a los postulados psicoanalíticos, los cuales volcaron la atención sobre rasgos ocultos de la intimidad, los afectos y la emocionalidad ${ }^{47}$. De modo que el individuo asalariado estaría caracterizado, presuntamente, por una nerviosidad casi inherente a su condición, además de estar aquejado por un intenso complejo de inferioridad ${ }^{48}$. Así, pues, Restrepo, Jaramillo, Valencia y Rosie (1968), se apoyaron en un corpus teórico desarrollado por ciertos autores, como el político socialista y profesor de psicología social, Herman de Man, en su texto Au-delá du marxisme ${ }^{49}$, el psicoterapeuta Alfred Adler, con su texto Le temperament nerveux ${ }^{50}$, Emmanuel Moinier y su
Traité du caractere ${ }^{51}$; Jean Lacroix, Force et faiblesses de la famille ${ }^{52}$; E de Greef, Notre destineé et nos instincts $^{53}$. Recurrir a categorías conceptuales como proletario, fuerza de trabajo y marxismo para referirse a las carencias mentales de los trabajadores, revelaba en este caso un cierto conocimiento del vocabulario marxista.

Ello también supuso una visión alarmista, desde lo psicológico, respecto a la situación misma del trabajador colombiano a partir de la segunda mitad del siglo $X X$, debido a su fuerte potencial organizativo. A modo de ilustración, Jaramillo, Valencia y Rosie (1968), señalaban que, en una calle cualquiera, una mujer asalariada tendría más razón de volverse nerviosa que la esposa de un alto funcionario, en virtud de sus inhibiciones y complejos. Incluso en una conversación casual, el asalariado, en general, se irritaría con mayor facilidad por las desigualdades percibidas, mientras que los demás no las notarían. En este caso, la susceptibilidad frente a la desigualdad, aceptada por estos autores en 1968, se constituyó en un signo clínico de nerviosidad y de múltiples traumas. Desde aquel momento estaría en capacidad de proyectar a las figuras de autoridad como el capataz, el burgués, el sacerdote, los ricos y políticos, su dolorosa oposición a la soberanía paterna ${ }^{54}$. La libertad entrañaría una "emancipación del padre" y el ideal de igualdad escondería la necesidad de una relación de hermanos. El egoísmo, el instinto de conservación, el ideal de igualdad y de dignidad humana, la voluntad de poder y de compensación de traumas infantiles, la necesidad de liberarse de la autoridad severa de los padres, en fin, la agresividad contra toda autoridad bajo el ideal de la "fraternidad democrática" ${ }^{55}$, se erigieron en una nueva armadura sintomatológica que fungió como correlato psíquico para deslegitimar cierto tipo de efervescencia social de la época, diferente al innatismo de comienzos de siglo.

La naturalización de las desigualdades, la responsabilidad individual y la omisión de los múltiples condicionantes sociales, se vieron acreditadas, en este caso, por una verdad sobre la biografía del individuo en su recorrido vital y construido a la luz del registro psicológico. Si bien se le reconocía al proletario el sentimiento de la igualdad y de la defensa de los derechos del hombre, detrás de ello, según este tipo de principios, se abría paso una fuerza primitiva mucho más peligrosa y oscura. Así, pues, no solo la psicologización de los trabajadores se orientó a señalar los presuntos defectos emocionales e inclinaciones 
políticas, sino que, desde el ámbito estatal, Jueces Militares y el Ministerio de Trabajo comenzaron a ejercer presión sobre los cuadros sindicales (Castaño, 2015a, p. 345). Estas expresiones reivindicativas permiten considerar que la patologización de ciertas conductas respondió a un entorno crecientemente conflictivo en las relaciones obrero-patronales, en el que se esperaba que este tipo de saberes diesen una explicación satisfactoria y con pretensiones científicas a estos fenómenos. Se pretendía que dicha explicación causal sobre un panorama convulso en lo social y laboral, contribuyera a sentar las bases para un tipo de intervención psicológica que neutralizara los conflictos de clase.

\section{CONSIDERACIONES FINALES}

Lo examinado hasta ahora permite vislumbrar dos fenómenos en relación con las formas de comprender el "problema" del trabajador durante el periodo analizado. En primer lugar, es posible identificar un proceso de objetivación de lo mental desde el campo médico, en relación con enfermedades como la neurastenia y la fatiga. Dicho proceso tuvo como finalidad examinar los efectos acarreados por el trabajo intelectual de un sector poblacional bastante minoritario para la época. En segundo lugar, el proceso de psicologización experimentado durante la segunda mitad del siglo marcó distancia respecto de las viejas corrientes ligadas al movimiento higienista, el movimiento de las ciencias morales y el movimiento de los grandes alienistas (Dejours, 2001, p. 208). No se trataba de unos principios epistémicos herederos de Eugene Buret, con sus reflexiones sobre la miseria de las clases laboriosas durante el siglo XIX, ni de Louis René Villermé (1971), quien, por intermedio de la Academia de Ciencias Morales de París, había trazado el camino en torno a las reflexiones sobre las miserias materiales y morales de los obreros (Perrot, 2011, p. 204). Ni siquiera se hace referencia a las nosografías y etiologías ligadas a las amenazas de la degeneración (propias de la primera mitad del siglo XX), cuya función se centró en el anhelo de protección para evitar así la decadencia de las poblaciones más pobres (Foucault, 2000, p. 294).
Todo ello, en síntesis, permitió examinar de qué manera la neurastenia comenzó a ser objetivada desde la medicina, no solo como efecto patológico de la civilización, sino también como consecuencia de los trabajos intelectuales desarrollados a comienzos del siglo veinte, entre los cuales sobresalían escritores, periodistas, sacerdotes, entre otros. De igual modo, el análisis de la astenia y la fatiga acarrearon una paulatina extensión de los padecimientos mentales sobre otras categorías ocupacionales, como los obreros. Lo anterior reveló un fenómeno ligado con la ampliación, no solo de dichas categorías ocupacionales, sino también del aparato productivo entre la primera y la segunda mitad del siglo XX. Se pudo constatar cómo la neurastenia y la fatiga se constituyeron en unas categorías destinadas a objetivar unas presuntas averías mentales que aquejaban a un sector laboral minoritario.

En ese sentido, la astenia de los obreros y la fatiga se enfocaron en el análisis de los impactos mentales ocasionados por el trabajo físico desempeñado por un sector poblacional, ubicado en la base de la pirámide social. A su vez, el tránsito de la medicalización a la psicologización de lo mental permitió constatar una relación cada vez más estrecha entre el ámbito laboral con la esfera privada de los trabajadores de ambas ciudades. De acuerdo con lo desarrollado en este artículo, es posible plantear una última consideración. La irrupción de ese proceso de psicologización (a diferencia de la medicina "somática" de la primera mitad del siglo XX) pretendió funcionar como un mecanismo sofisticado de intervención y encauzamiento sigiloso sobre los diferentes planos de la esfera personal y laboral. Lo anterior revela cómo la reacción frente a la desigualdad social y laboral, se tornó en un importante elemento de cautela y prescripción por parte de este tipo de discurso "psi". Las inmensas expectativas frente a lo ofrecido por el desarrollo industrial en Colombia, especialmente en Bogotá y Medellín, desviaron la crítica del complejo marco social, para centrar la atención sobre la psique de los individuos, su intimidad y su presunta incapacidad "patológica" para amoldarse al rol de un obrero y trabajador dócil. 


\section{NOTAS}

1 Este artículo es el resultado de una tesis doctoral en Historia desarrollada en la Universidad Nacional de Colombia, sede Medellín, titulada Cuerpos y almas para el trabajo: la psicologización de los trabajadores en Bogotá y Medellín, 1928-1994.

2 El 31 de marzo de 1994 y, a través del artículo 10 de la resolución 1016, se legisló en materia de Medicina Preventiva y del Trabajo. Sin embargo, fue con el decreto 1832 de 1994 cuando se incluyeron las patologías causadas por el estrés en la tabla de enfermedades laborales.

3 Rueda, Prospero, “El determinismo cederá el paso a la psicología", en Temas, Medellín, enero de 1944, p.37.

4 Mantegazza, Pablo, El siglo de los nervios, Bogotá, Imprenta de la Luz, 1888, p.5.

5 Mantegazza..., p.7.

6 Mantegazza...p.21.

7 Rodríguez Piñeres, Julio, Neurastenia, Bogotá, Imprenta y librería de Medardo Rivas, Bogotá, Tesis presentada para el doctorado en medicina y cirugía, 10 de noviembre de 1898.

8 Rodríguez Piñeres..., p.15.

9 Delgado C, Jaime, Histeria en el ejército, Bogotá, Tesis para el doctorado en medicina y cirugía, Bogotá, Imprenta eléctrica, 1910.

10 Rodríguez Piñeres Neurastenia, Bogotá, Imprenta y librería de Medardo Rivas, Bogotá, Tesis presentada para el doctorado en medicina y cirugía, 10 de noviembre de 1898. p.80.

11 Rodríguez Piñeres..., p.11.

12 María Calle, Miguel, "La astenia de los obreros I", en La organización, Medellín, 1910, pp.1-2.

13 María Calle, Miguel..., p.1.

14 María Calle, Miguel..., p.2.

15 María Calle, Miguel..., p.2.

16 Mantegazza, Pablo, El siglo de los nervios, Bogotá, Imprenta de la Luz, 1888.

17 María Calle, Miguel María Calle, Miguel, "La astenia de los obreros I", en La organización, Medellín, 1910, pp.1-2.

18 Uribe, Basilio, "Neurosis", en Alpha, Medellín, 2, 13, enero de 1907, p.340.

19 Anónimo, "Algunas enfermedades", en Temas, Medellín, noviembre de 1942, p.890.

20 Anónimo, "El trabajo ahuyenta los males", en El Sol, Medellín, 1914, p.1.

21 Anónimo..., p.1.

22 Anónimo..., p. 1.

23 Mejía, Braulio, "Los estados nerviosos", en Boletín Clínico, Medellín, 1, mayo de 1932, p.203.

24 “Algunas enfermedades", en Temas, Medellín, 3, 15-20, julio-diciembre de 1942, p.890.
25 Isaza, Juan, Contribución al estudio de la fiebre biliosa hemoglobinúrica, Tesis para el doctorado en Medicina y $\mathrm{Ci}$ rugía, Medellín, Facultad de Medicina y Ciencias Naturales, Universidad de Antioquia, 1920, p.45.

26 Ortiz Velásquez, Julio, "Psicastenia y tisifobia", en Boletín Clínico. Órgano de la Facultad de Medicina y Ciencias Naturales de la Universidad de Antioquia, 9, 1, Medellín, febrero de 1936.

27 “Algunas enfermedades", en Temas, Medellín, 3, 15-20, julio-diciembre de 1942, p.890.

28 Osorio Lizarazo, J A, Hombres sin presente. Novela de Empleados Públicos, Bogotá, Editorial Minerva, 1937, p.99.

29 Morales B, Emilio, Algunas consideraciones sobre la fisiología industrial, la fatiga y accidentes de trabajo, Tesis para optar al título de doctor en medicina y cirugía, Facultad de Medicina, Universidad Nacional, Bogotá, 1937, p.36.

30 Morales, Emilio..., p.38.

31 Morales, Emilio..., p.40.

32 De Madariaga, César, Introducción al estudio del factor humano en la industria. Nociones de psicoeconomía, Bogotá, 1946, p.97.

33 Marín, Juan, "La preocupación anula la capacidad de trabajo", en Temas, Medellín, agosto de 1944, p.143.

34 Vasco Gutiérrez, Eduardo, "Existe un tratamiento de la oligofrenia", en Anales de la Academia de Medicina de Medellín, Medellín, 3, 6, oct-sept de 1948, p. 391

35 Vasco Gutiérrez, Eduardo..., p. 391.

36 Para Eduardo Vasco, basándose en la definición establecida por la British Mental Deficiency Act, la oligofrenia era un estado en el cual el desarrollo mental había quedado detenido e incompleto, presentándose antes de los 18 años. Dicha enfermedad se presentaría, ya fuese por causa de condiciones innatas o en razón a enfermedades o traumatismos. El oligofrénico sería definido como un sujeto que nació con una deficiencia en su vida mental, debida, presuntamente, a que su cerebro no pudo alcanzar un completo desarrollo., p.391.

37 Anónimo, "El nuevo mal del siglo: la fatiga" en Cromos, Bogotá, 2214, noviembre 16, 1959, p.25.

38 Anónimo..., p.26.

39 "Apuntes médicos: nuestra actitud hacia el trabajo" El Correo, Medellín, febrero 27 de 1968, p.13.

40 Anónimo, "Tiempo es oro", en Lógica y trabajo, Medellín, noviembre de 1945, p.121.

41 Anónimo..., p.122.

42 Macrodiagnóstico de Salud Mental en Colombia en 1973, Ministerio de Salud Pública. Dirección de atención médica. División de la salud mental, Bogotá, 1974, p.3.

43 Anónimo, "Psiquiatría en la productibilidad colombiana", en Revista Colombiana de Psiquiatría, Bogotá, 1., 5, agosto de 1966, p.217. 
44 Restrepo José Miguel, "predisposición en las enfermedades" en Orientaciones médicas, Medellín, 7., 5, mayo de 1958, pp.243-252.

45 Uribe Cualla, Guillermo, "Aspectos psiquiátricos de los inmigrantes", en Anales neuropsiquitricos, Bogotá, 10., 3940, pp.61-68.

46 Restrepo José Miguel, "Medicina Industrial" en Orientaciones médicas, Medellín, 13., 7, Julio de 1959, p.275.

47 Jaramillo, Luis Gabriel, Valencia Enrique, Rosie, Ireneo, "Temas de psicología: los complejos y reacciones en el alma del proletario" en Revista Colombiana de Psiquiatría, Bogotá,1., 8, abril de 1968, p.623.

48 Jaramillo, Luis Gabriel, Valencia Enrique, Rosie, Ireneo, "Temas de psicología: los complejos y reacciones en el alma del proletario" en Revista Colombiana de Psiquiatría, Bogotá,1., 8, abril de 1968, p.623.

49 De Man, Henrman, Au delá du marxisme, Paris, Seuil, 1974.

\section{BIBLIOGRAFÍA}

Archila Neira, Mauricio (2003a), Idas y venidas, vueltas y revueltas: protestas sociales en Colombia, 1958-1990, Bogotá, Instituto Colombiano de Antropología e Historia, Cinep, p. 508.

Archila Neira, Mauricio (2003b), Cultura e identidad obrera. Colombia 1910-1945, Bogotá, Cinep, p. 475.

Le Bianic, Thomas (2004), "Le Conservatoires des arts et Métiers et la "machine humanine Naissance et développement des sciences de L'homme au travail au CNAM (1910-1990)", Revue d'Historie des Sciences Humaines, 2 (11), pp. 185-214.

Billiard, Isabelle (2002), "Les Peres fondateurs de la psychopathologie du travail en butte a I' enigme du travail", Cliniques Méditerranéenes, 2 (66), pp. 11-29.

Canguilhem, Georges (1986), Lo normal y lo patológico, México D.F, Siglo XXI, p. 242.

Caponi, Sandra (2015). Del culto a la laboriosidad a la preocupación por la fatiga: dos miradas sobre la pobreza (Argentina, 1903-1904), Revista Mundos do Trabalho. (Florianopolis), 7 (13), pp. 43-64

Castaño González, Eugenio (2015a), Fisonomías del miedo: un paulatino enmudecimiento. Recuento de luchas y lógicas de la violencia antisindical en el departamento del Atlántico: CUT, Sintraelecol y Anthoc. 1975-2012, Medellín, Editorial Escuela Nacional Sindical, p. 345.

Castaño González, Eugenio (2015b), Nos hacen falta. Memoria Histórica de la violencia antisindical en Antioquia, Atlántico y Santander (1975-2012), Medellín, Escuela Nacional Sindical, p. 482.

Castro, Santiago (2009), Tejidos Oníricos. Movilidad, capitalismo y biopolítica en Bogotá (1910-1930), Bogotá, Pontificia Universidad Javeriana, p. 274.
50 Adler, Alfred, Le temperament nerveux. Éléments $d$ une psychologie individualle et application a la psychotérapie, Quebec, Université du Québec a Chicoutimi, (s/f). Consultar en el siguiente enlace: http://classiques.uqac.ca/classiques/adler_alfred/temperament_nerveux/adler_temperament_nerveux.pdf

51 Moinier, Emmanuel, Traiter du caractere, Paris, Seuil, 1974.

52 Lacroix, Jean, Force et faiblesses de la famille, Paris, Seuil, 1965.

53 De Greef, E, Notre destineé et nos instincts, Librairie Plon, 1943.

54 Jaramillo, Luis Gabriel; Valencia Enrique, Rosie Ireneo, "Temas de psicología: los complejos y reacciones en el alma del proletario" en Revista Colombiana de Psiquiatría, Bogotá, 1, 8, abril de 1968, p.626

55 Jaramillo, Luis Gabriel; Valencia Enrique, Rosie Ireneo, "Temas de psicología: los complejos y reacciones en el alma del proletario" en Revista Colombiana de Psiquiatría, Bogotá, 1, 8, abril de 1968, p.626.

Dejours, Cristophe (2001), Trabajo y desgaste mental. Una contribución a la psicopatología del trabajo, Buenos Aires, Grupo Editorial Lumen, p. 208.

Foucault, Michel (2000), Los anormales, Buenos Aires, Fondo de Cultura Económica, p. 294.

Gallo, Oscar (2016a), Trabalho, medicina e legislacao na Colombia (1919-1946), Tesis de doctorado, Universidade Federal de Santacatarina, Florianopolis,

Gallo, Oscar (2016b), “Luz sobre el fenómeno de un corazón aparentemente infatigable. La fatiga de la clase obrera en Colombia. 1898-1946". En: Gallo, Oscar y Castaño, Eugenio (Eds.), Salud laboral en el siglo XX y XXI. De la negación al derecho a la salud y la enfermedad. Medellín, Escuela Nacional Sindical, pp. 219-252

Gallo, Oscar y Ramacciotti, Karina (2019), "Medicina del trabajo y Seguridad Social: una mirada desde Latinoamérica", $D y$ namis, 39 (2), pp. 279-288, p. 6.

Hacking, lan (2006), La domesticación del azar. La erosión del determinismo y el nacimiento de las ciencias del caos, Barcelona, Editorial Gedisa, p. 242.

Haidar, Victoria (2016), “¡Buscad al hombre!: la problematización del factor humano en la explicación de los accidentes de trabajo. Argentina, entre la actualidad y la historia". En: Gallo, Oscar y Castaño, Eugenio (Eds.), La salud laboral en el siglo XX y XXI. De la negación al derecho a la salud y la enfermedad, Medellín: Escuela Nacional Sindical.

Hopenhayn, Martín (2001), Repensar el trabajo. Historia, profusión y perspectivas de un concepto, Buenos Aires, Norma, p.149.

Jaraba, Bruno (2014), Un escritorio para Mercedes: revisando el mito fundacional de la psicología en Colombia, Tesis de 
maestría en sociología, Universidad Nacional de Colombiasede Bogotá.

Kalmanovitz, Salomón (2015), Breve historia económica de Colombia, Bogotá, Fundación Universidad de Bogotá Jorge Tadeo Lozano, p. 245.

Mora, Alfonso (1949), “Escuelas de trabajo. El problema del proletariado", Progreso, p.4

Motta, Julia María (2008), "Fragmentos da historia e da memoria da psicologia no mundo do trabalho no Brasil: relações entre a industrialização e a psicologia", Tesis de doctorado en Salud Colectiva, Universidade Estadual de Campinas.

Ocampo Gaviria, José Antonio; Bernal, Joaquin; Avella, Mauricio y Errázuriz, María (1987), "La consolidación del capitalismo moderno (1945-1986)". En: Ocampo Gaviria, José Antonio (Ed.), Historia económica de Colombia, Bogotá, Siglo XXI Editores, pp. 128-162.

Ouvrier, Régis (2010), “L'histoire des chaires du CNAM concernant l'Homme au travail (1900-1945) entre production de savoirs et engagement politique", Les Cahiersd 'historie, Revue d' historie critique, 111, pp. 99-122.

Perrot, Michelle (2011), Historia de las alcobas, México D.F, Fondo de Cultura Económica, p.204.

Pohl-Valero, Stefan (2009), "La comunicación de la termodinámica. Física, cultura y poder en la España de la segunda mitad del siglo XIX", Memoria y Sociedad, 13(27), pp. 121-142.

Pohl-Valero, Stefan (2010), "La termodinámica, pensamiento social y biopolítica en la España de la restauración", Universitas Humanistica, (69), pp. 36-60.

Pohl-Valero, Stefan (2014), "La raza entra por la boca": Energy, Diet, and Eugenics in Colombia, 1890-1940", Hispanic American Historical Review, 94 (3), pp. 455-486.

Quetelet y Durkheim (2008), Las reglas del método sociológico, Bogotá, Skala.

Rabinbach, Anson (1990), The human motor. Energy, fatigue, and the origins of modernity. Berkeley, University of California Press, p. 432.

Rabinbach, Anson (1996), "Neurastenia y modernidad", Biblioteca de México, (31), pp. 38-42.

Roldán, Diego (2010), "Discursos alrededor del cuerpo, la máquina, la energía y la fatiga: hibridaciones culturales en la
Argentina de fin-de-siecle", História, Ciencias, Saúde-Manguinhos, 17 (3), pp. 643-661.

Rose, Nikolas (1990), Governing the soul. The shaping of the private self, London and New York, Routledge, p. 352.

Roselli Cock, Diego Andrés; Otero, Andrés y Heller Mitrani, Daniella (2000), La medicina especializada en Colombia. Una aproximación diagnósitica, Bogotá, Centro Editorial Javeriano, p. 224.

Roselli, Humberto (1968), Historia de la psiquiatría en Colombia, Tomo I, Bogotá, Editorial Horizontes.

Ruperthuz, Mariano (2019), La recepción de la Higiene mental en Chile: prevenir la locura en un contexto de modernización y aceleración del tiempo en la ciudad de Santiago, 19001954, Tesis de doctorado en Historia, Universidad de Santiago de Chile.

Sánchez, Dayanna (2009), "Incidencia del modelo agroexportador en la construcción regional e identitaria colombiana", Criterios: Cuadernos de ciencias jurídicas y Política Internacional, 2 (2), p. 49-65.

Sánchez, Ricardo (2009), iHuelga! Luchas de la clase trabajadora en Colombia, 1975-1981, Bogotá, Universidad Nacional de Colombia, p. 480.

Saraceno, Marco (2013), De la mesure du corps a la politique des corps: une historie des sciences du travail 1880-1920, Tesis de doctorado en Sociología, Université de Nanterre, París.

Solano, Sergio Paolo (2011), "Trabajo, raza, ciudadanía y estilos de vida en Colombia durante el siglo XIX", Revista de Ciencias Sociales, 17 (2), pp. 341-356.

Vásquez, María Fernanda (2015), Degenerados, criminosos e alienados. Para uma história do conceito de degeneracao na Colombia, 1888-1950, Tesis de doctorado, Universidade Federal de Santa Catarina, Florianopolis.

Vatin, François (2004), Trabajo, ciencias y sociedad. Ensayos de sociología y epistemología del trabajo, Buenos Aires, Lumen Humanitas, p. 244.

Villegas, Álvaro (2014), "El valle del río Magdalena en los discursos letrados de la segunda mitad del siglo XIX: territorio, enfermedad y trabajo", Folios, (39), pp. 149-159.

Villermé, Louis René (1971), État physiqué et moral des ouvriers, Paris, Union générale d'Editions. 